\title{
Integrating supervised consumption into a continuum of care for people who use drugs
}

\author{
Ayden Scheim PhD, Dan Werb PhD
}

Cite as: CMAJ 2018 August 7;190:E921-2. doi: 10.1503/cmaj.180824

$\mathbf{T}$ he opioid overdose crisis in North America demonstrates a need to scale up supervised-consumption services, as well as to experiment with a mix of potential service models. A range of new-to-Canada models have been implemented over the past two years. In some cities, including Toronto and Ottawa, health authorities initially focused on embedding the services within community health agencies to provide a continuum of health care and treatment for substance use disorders, but there are no data on the effectiveness of this model. With an increasing diversity of models of supervisedconsumption services in operation, there exists a window of opportunity for a second generation of research in this area that moves from asking whether such services are effective in reducing drug-related harms - which we know them to be ${ }^{1}-$ to asking whether, how and under what conditions their benefits can be maximized.

Models of supervised-consumption services operating in Canada include peer-run "overdose-prevention sites," stand-alone storefronts, mobile vans, co-location with harm-reduction programs or social housing, in-hospital services, women-only sites and the aforementioned integrated model. Some of these operate within community health centres that also offer services for populations that do not use drugs. The plurality of models raises questions as to their relative effectiveness. These questions are not answered by the existing scientific literature, which is dominated by reports from two stand-alone sites in Vancouver and Sydney, Australia, employing comparable models. ${ }^{1}$ The evidence base on alternative models is insufficient to guide policy in this area, and it would be premature to consider service models interchangeable. Nevertheless, officials in Seattle recently announced they will operate a mobile supervised-consumption service because no fixed location could be secured.

Insite, Canada's first formal supervised-consumption service, opened in Vancouver in 2003 and remained the only sanctioned site in Canada for 12 years. ${ }^{2}$ Insite is a stand-alone, storefront model that offers basic nursing care (including initial prescriptions for opioid agonist therapy) and a small co-located medically supervised detoxification facility. Extensive evaluation has established Insite's public health benefits. ${ }^{1}$ However, it is also evident that stand-alone models have limited reach. ${ }^{3}$ They serve a small geographic area (the distance clients will walk), and even

\section{KEY POINTS}

- Supervised-consumption services are known to be effective in reducing drug-related harms, and several dedicated services now exist in Canada.

- It is now time to move from asking whether such services are effective to asking whether, how and under what conditions their benefits can be maximized.

- Integrated and co-located health service models - effectively "one-stop shops" - could improve health outcomes for people who inject drugs by combining the prevention of immediate drug-related harms with access to primary care, mental health care and social service programs.

among regular service users, only a proportion of injections are covered (43\% of Insite users accessed the site for fewer than onequarter of injections ${ }^{4}$ ). Moreover, stand-alone models attract a highly socially vulnerable population, ${ }^{5}$ and clients continue to face disparities in health status, homelessness and access to opioid agonist therapy. ${ }^{6}$ This suggests a need for greater scale-up of existing models as well as for new models that offer a larger suite of on-site health services.

Integrated and co-located health service models have been developed to address HIV and hepatitis C epidemics among people who inject drugs, ${ }^{7}$ and have been successful in improving the quality of client care. ${ }^{8}$ Integrated services offer clients "one-stop shopping" in settings where they have established trusting relationships, in contrast to the stigma and mistrust that often characterize other health care encounters. ${ }^{7,8}$ Although stand-alone supervised-consumption services have demonstrated some success in making referrals to external health services, ${ }^{1}$ referrals to co-located services are likely to be more successful given the difficulties their client populations typically face in accessing medical care. ${ }^{9}$ As perhaps the lowest-barrier health services available to people who use drugs, supervised-consumption services represent an ideal fulcrum for service integration.

Such integration is occurring within community health centres in Toronto and Ottawa, where supervised-consumption services aim to prevent immediate drug-related harms while connecting clients to existing on-site primary care, mental health care and social service programs. These integrated models also 
offer opportunities for innovative service delivery to fill gaps in the current system of care. In Toronto, Ottawa and Vancouver, drug-checking programs using spectrometry are being put in place within supervised-consumption services so that detailed information on the composition of street drugs can be disseminated in an effort to reduce overdose risk. One Toronto site, The Works, already includes an on-site methadone and suboxone treatment clinic. Further, there is increasing interest across Canada in managed opioid programs to divert patients from adulterated street drugs and to offer managed care for opioid use disorder. Integrated supervised-consumption services are being considered as natural hubs for such programs.

Despite the theoretical advantages of integrated models of supervised-consumption services, there are also potential challenges requiring community-engaged investigation. For example, there is tension between integration with clinical care and provision of low-barrier supervised-consumption services. For some individuals who use drugs, the regulations imposed by government-sanctioned models (e.g., time limits and inability to share drugs) are unacceptable, ${ }^{3,10}$ and others may prefer to access supervised-consumption services separately from other health services to maintain their anonymity. Such individuals can choose to access lower-threshold overdose prevention sites in some cities, but these are ultimately intended to be temporary solutions. The impacts of integrated models may also be constrained by operational restrictions of health care facilities, such as limited hours of operation. Therefore, we propose that a consideration of the relative benefits of stand-alone, integrated and low-threshold models should be prioritized in second-generation research on supervised-consumption services, particularly with respect to their appropriateness for urban, suburban, rural and remote communities, and for specific subpopulations, such as women and Indigenous people.

Numerous challenges limit implementation of supervisedconsumption services, even during this period of heightened mortality from opioid overdose. Municipalities need not choose from a single model, and all options should be on the table, from peer-run through clinically embedded supervised-consumption services. Indeed, the operation of diverse service models across multiple sites within a given city is likely optimal. The social contexts, needs and preferences of people who use drugs across settings are diverse, and so too must be the public health response. At the same time, precisely because supervised-consumption services can be challenging to establish, efforts should be made to understand when, why and for whom various models are most beneficial, rather than treating them as interchangeable. Specifically, implementing and evaluating programs that integrate supervised consumption within a broader spectrum of care may reduce substantial barriers to progression along the continuum of treatment for substance use disorder for marginalized individuals who use drugs, filling an ongoing gap in care that contributes to the worsening of Canada's opioid overdose crisis.

\section{References}

1. Kennedy MC, Karamouzian M, Kerr T. Public health and public order outcomes associated with supervised drug consumption facilities: a systematic review. Curr HIV/AIDS Rep 2017;14:161-83.

2. Kerr T, Mitra S, Kennedy MC, et al. Supervised injection facilities in Canada: past, present, and future. Harm Reduct J 2017;14:28.

3. Kerr T, Small W, Moore D, et al. A micro-environmental intervention to reduce the harms associated with drug-related overdose: evidence from the evaluation of Vancouver's safer injection facility. Int J Drug Policy 2007;18:37-45.

4. Stoltz J-A, Wood E, Small W, et al. Changes in injecting practices associated with the use of a medically supervised safer injection facility. J Public Health (Oxf) 2007;29:35-9.

5. Wood E, Tyndall MW, Li K, et al. Do supervised injecting facilities attract higherrisk injection drug users? Am J Prev Med 2005;29:126-30.

6. Wood E, Tyndall MW, Qui Z, et al. Service uptake and characteristics of injection drug users utilizing North America's first medically supervised safer injecting facility. Am J Public Health 2006;96:770-3.

7. Sylla L, Bruce RD, Kamarulzaman A, et al. Integration and co-location of HIV/ AIDS, tuberculosis and drug treatment services. Int J Drug Policy 2007;18:306-12.

8. Bachireddy C, Soule MC, Izenberg JM, et al. Integration of health services improves multiple healthcare outcomes among HIV-infected people who inject drugs in Ukraine. Drug Alcohol Depend 2014;134:106-14.

9. Bruggmann P, Litwin AH. Models of care for the management of hepatitis $\mathrm{C}$ virus among people who inject drugs: One size does not fit all. Clin Infect Dis 2013; 57(Suppl 2):S56-61.

10. Small W, Shoveller J, Moore D, et al. Injection drug users' access to a supervised injection facility in Vancouver, Canada: the influence of operating policies and local drug culture. Qual Health Res 2011;21:743-56.

Competing interests: Dan Werb received funding from Health Canada to implement drug-checking services within supervised-consumption sites in Toronto. None declared by Ayden Scheim.

This article has been peer reviewed.

Affiliations: Centre on Drug Policy Evaluation (Scheim, Werb), Li Ka Shing Knowledge Institute, St. Michael's Hospital, Toronto, Ont.; Division of Infectious Diseases and Global Public Health (Scheim, Werb), University of California San Diego School of Medicine, La Jolla, Calif.; Institute of Health Policy, Management and Evaluation (Werb), University of Toronto, Toronto, Ont.

Contributors: Both authors drafted the manuscript, gave final approval of the version to be published and agreed to be accountable for all aspects of the work.

Funding: Ayden Scheim is supported by the Pierre Elliott Trudeau Foundation and a Canadian Institutes of Health Research (CIHR) Fellowship. Dan Werb is supported by a CIHR New Investigator Salary Award.

Correspondence to: Ayden Scheim, ayden@cdpe.org 